\title{
A Novel Approach for Multiple Moving Target Localization Using Dual-Frequency Radars and Time-Frequency Distributions
}

\author{
Yimin Zhang, Moeness Amin, and Fauzia Ahmad \\ Radar Imaging Lab, Center for Advanced Communications \\ Villanova University, Villanova, PA 19085 \\ E-mail: \{yimin.zhang, moeness.amin, fauzia.ahmad\}@villanova.edu
}

\begin{abstract}
Accurate range estimation and tracking of moving targets is an important task in urban sensing applications. A dual-frequency radar, which estimates the range of a target based on the phase difference between two closely spaced frequencies, has been shown to be a cost-effective approach to accomplish both range-to-motion estimation and tracking. This approach, however, suffers from two drawbacks: it cannot deal with multiple moving targets, and has poor performance in noisy environments. To overcome these drawbacks, we propose, in this paper, the use of timefrequency signal representations. High signal-to-noise ratio (SNR) is obtained by focusing on the moving target instantaneous Doppler frequency law provided by the power localization properties of time-frequency distributions. The case of multiple moving targets is handled by separating the different Doppler signatures prior to phase estimation.
\end{abstract}

\section{INTRODUCTION}

Through-the-wall radar imaging (TWRI) and sensing is an emerging technology supporting a range of civilian and military applications [1-6]. TWRI has been recently sought out for surveillance and reconnaissance in urban environments, requiring not only the layout of the building, including types and locations of walls, but also detection and localization of both moving and stationary targets within enclosed structures. This technology can also be used by firefighters to detect and locate survivors, by criminal justice officers for enhanced situational awareness and tailored tactical operations, and in search and rescue operations in natural disasters.

There are many challenges facing the development of a successful TWRI system to meet the needs of urban sensing. The system should be low cost, light weight, reliable, portable, and user-friendly. It should be able to detect, locate, and classify a variety of indoor targets, including both moving and stationary objects, enclosed in small spaces and in the presence of significant multipath propagation and heavy clutter. Doppler radars satisfy all of the above conditions, specifically when the location of moving targets, rather than target resolutions, are of primary interest.

This work was supported in part by ONR under grant N00014-07-10043 and in part by DARPA under contract no. HR0011-06-C-0110. The content of the information does not necessarily reflect the position or the policy of the Government, and no official endorsement should be inferred. Approved for Public Release, Distribution Unlimited.
In this paper, we consider Doppler radars for both target motion detection and ranging. Doppler radars typically use a single frequency and, as such, suffer from large range ambiguity. In urban sensing applications, range ambiguity may be eliminated if the difference between consecutive range estimates is larger than the building dimensions. Due to restrictions on antenna size and frequency allocation management, lowering the radar frequency to reduce the range ambiguity is not a viable option for urban sensing applications. A simple and low cost alternative is the dualfrequency continuous-wave $(\mathrm{CW})$ radar based approach, which employs two different frequencies and simultaneously measures the phase change with respect to time, for each of the two frequencies, separately [7-9]. It uses phase comparison of the reflected signals to estimate the range, whereas the target velocity is directly obtained from the Doppler shift. The two carrier frequencies, and hence their respective wavelengths, determine the maximum unambiguous range of the target. The method can detect moving targets up to a maximum range corresponding to $360^{\circ}$ phase difference of the radar returns for two distinct carrier frequencies. This can significantly increase the unambiguous range compared to that of single frequency operation.

The performance of the dual-frequency radar technique rapidly degrades when operating in a noisy cluttered environment [10], which is commonly encountered in urban sensing applications, and when dealing with multiple targets. In the latter, the received signal is the superposition of returns from different targets and, therefore, phase information corresponding to individual targets cannot be obtained from the raw data. Similarly, when there are strong multipaths of a single or multiple targets, the phase is distorted and the phase information from raw returns does not yield correct range estimates.

In this paper, we propose the use of time-frequency representation of the target return signals for improved range estimation and tracking of one or more moving targets. The proposed technique has three major advantages. First, the time-frequency representation allows concentration of the signal energy around the instantaneous Doppler frequency and thus enhances the signal-to-noise ratio (SNR) [11-12]. Second, time-frequency representations provide a platform to obtain enhanced phase information as well as the Doppler signatures of the targets. Thus, range estimation and tracking can be improved through the fusion of Doppler signature and the phase information, which is most effective, particularly in low 
SNR scenarios. Third, separation of multiple target signals can be accomplished in the time-frequency domain.

\section{RANGE ESTIMATION USING DUAL- FREQUENCY CW RADAR}

Consider a dual-frequency $\mathrm{CW}$ radar operating at frequencies $f_{1}$ and $f_{2}$. The baseband radar return at frequency $f_{i}, i=1,2$, can be expressed as,

$$
s_{i}(t)=\rho_{i}(t) \exp \left(-j \phi_{i}(t)\right), \quad i=1,2,
$$

where $\rho_{i}(t)$ and $\phi_{i}(t)$ are, respectively, the range- dependent amplitude and the phase of the return corresponding to the $i$-th frequency of operation. If $R(t)$ is the law of motion of the target, then

$$
\phi_{i}(t)=\frac{4 \pi f_{i} R(t)}{c}, \quad i=1,2 .
$$

The Doppler frequency shift, $f_{D, i}(t)$, is the differential of the corresponding phase, $\phi_{i}(t)$, and is given by

$$
f_{D, i}(t)=-\frac{1}{2 \pi} \frac{d \phi_{i}(t)}{d t}=-\frac{2 f_{i}}{c} \frac{d R(t)}{d t}, \quad i=1,2 .
$$

If both phases are measured modulo $2 \pi$, then

$$
\phi_{1}(t)=\frac{4 \pi f_{1} R(t)}{c}+2 n \pi, \quad \phi_{2}(t)=\frac{4 \pi f_{2} R(t)}{c}+2 m \pi,
$$

where $m$ and $n$ are unknown integers. Accordingly $[8,10]$

$$
\begin{aligned}
R(t) & =\frac{c}{4 \pi\left(f_{2}-f_{1}\right)}\left[\left(\phi_{2}(t)-\phi_{1}(t)\right)-2 \pi(m-n)\right] \\
& =\frac{c}{4 \pi\left(f_{2}-f_{1}\right)}\left(\phi_{2}(t)-\phi_{1}(t)\right)-\frac{c(m-n)}{2\left(f_{2}-f_{1}\right)} .
\end{aligned}
$$

The second term in the above equation induces ambiguity in range. For the same phase difference, the range can assume infinite values separated by $c /\left[2\left(f_{2}-f_{1}\right)\right]$. Therefore, the maximum unambiguous range is given by

$$
R_{\max }=\frac{c}{2\left(f_{2}-f_{1}\right)} .
$$

Depending on the application, sufficient unambiguous range can be achieved by properly selecting the frequency difference between the two carriers. For example, when frequencies of $1 \mathrm{GHz}$ and $990 \mathrm{MHz}$ are employed, the $10 \mathrm{MHz}$ difference in the carrier frequencies yields a $15 \mathrm{~m}$ unambiguous range. In urban sensing, this unambiguous range may be sufficient to uniquely solve for the target range, given prior knowledge of the structure bounds on target location. The bounds determine the integer value $m-n$ corresponding to the possible target range. The choice of the specific values of $f_{1}$ and $f_{2}$ can be made based on RF wall penetration and design issues of the radar units.

\section{TIME-FREQUENCY REPRESENTATION}

\section{A. Short-Time Fourier Transform}

It is clear from the previous section that the key to determining the range of a target is the estimation of the phase difference of the return signals corresponding to the two frequencies, $f_{1}$ and $f_{2}$. The successful application of the dual-frequency approach for range estimation of a single moving target has been demonstrated in $[8,10]$. However, as we discussed earlier, this method suffers from two fundamental problems, namely, poor performance in a noisy environment and the strict limitation that only a single moving target can exist in the scene. In this Section, we propose the use of timefrequency representation of the target return signals in conjunction with the dual-frequency approach to overcome these limitations.

In the following, we exploit the short-time Fourier transform (STFT) to demonstrate the effectiveness of timefrequency analysis to provide range estimates for multiple targets using a dual-frequency radar.

The STFT of a signal $x(t)$ can be defined as

$$
F_{x}(t, f)=\int_{-\infty}^{\infty} x(\tau) h(\tau-t) e^{-j 2 \pi f \tau} d \tau,
$$

where $h(t)$ is the window function. When no window is used, the STFT reduces to ordinary Fourier transform. The use of different windows allows trading-off the time and frequency resolutions. A larger window size provides higher frequency resolution at the expense of reduced time-resolution. In our analysis, we assume that the window span is sufficiently small such that each return signal has a linear phase (i.e., constant Doppler frequency) over the window time-duration. On the other hand, the window span is assumed to be large enough to achieve reasonable frequency resolution. Satisfactory time and frequency resolutions require proper selection of the sampling rate and window function.

Multiple target returns with distinct Doppler signatures can be separated in the time-frequency domain. Effective time-frequency representation tools, such as the magnitude square of (7), known as spectrogram, localize the signal power in the time-frequency domain. The target Doppler signature can, therefore, be obtained by locating the high power timefrequency peaks. It can be shown that, the phase information of a signal is preserved at the corresponding peak of the STFT signature [13]. With the ability to capture each Doppler signature of the moving targets in the scene, one can proceed to calculate the respective phase information and subsequently estimate the target range.

\section{B. Multipath Consideration}

Multipath is a common occurrence in a typical urban sensing environment. It is noted that the effect of multipath is very similar to the presence of another target. With a propagation environment with two or more paths, the phase of the received signal corresponds to neither the target (direct path), nor that of the indirect path.

The effect of multipath differs depending on the strength and location of the reflection. Because the phase of the combined signal is highly nonlinear with respect to the multipath strength, the phase of the combined signal is close to that of the target return signal if the multipath signal is weak compared to direct path return. Otherwise, the phase will be highly distorted and thus cannot render meaningful range information. 


\section{RANGE ESTIMATION}

\section{A. Range Estimation Based on STFT Phase Information}

As discussed earlier, this phase information, and thereby the range estimate, obtained from the time-frequency representation at the Doppler signatures is much more robust compared to that obtained from the raw data. This is due to the enhancement of the SNR when measured at the target instantaneous Doppler frequency.

The selection of the Doppler signatures in the timefrequency domain, in general, can be done using simple peak detection. With multiple moving targets, some additional work to trace different pieces of the Doppler signature to the same target is required. Separation of non-overlapping and crossing Doppler signatures can be effectively performed by observing the phase continuity [14], often describing motions of indoor animate or inanimate target.

\section{B. Range Estimation Based on Doppler Signatures}

In addition to the phase information, the Doppler signature of each target can also be obtained from the time-frequency representations. As a target moves, the phase progression can be obtained from the integral of the Doppler frequency, $f_{D, i}(t)$. As such, the phase information of the return signals and, subsequently, the ranges of moving targets, can be obtained from the Doppler signatures up to an initial value as follows,

$$
\hat{\phi}_{i}(t)=-2 \pi \int_{0}^{t} f_{D, i}(t) d t+\phi_{i}(0), \quad i=1,2,
$$

where $\phi_{i}(0)$ is an unknown initial phase at $t=0$. The unknown initial phase difference between the two carrier frequencies, denoted as $\phi(0)=\phi_{2}(0)-\phi_{1}(0)$, can be obtained by minimizing the overall distance between the Doppler signature based phase difference estimation $\hat{\phi}(t)=$ $\hat{\phi}_{2}(t)-\hat{\phi}_{1}(t)$ and that obtained at the selected STFT points $\widetilde{\phi}(t)=\widetilde{\phi}_{2}(t)-\widetilde{\phi}_{1}(t)$. That is,

$$
\phi(0)=\arg \min _{\phi(0)} \int\|\hat{\phi}(t)-\tilde{\phi}(t)\|^{2} d t,
$$

where the integral is evaluated over the entire observation period. Simulations and experiment results have shown that the range estimation based on the Doppler signature is much more robust to various perturbation factors caused by noises, reflection, and cross-component interference.

A Doppler signature bias may accumulate over time to yield a large error in the range estimate. Therefore, high resolution fast Fourier transform (FFT) operation in the STFT computation is desirable.

\section{Range Estimation Based on Bilinear Time-Frequency Representations}

The proposed method can also be straightforwardly extended to bilinear time-frequency representations, such as the Cohen's class of time-frequency distributions. For bilinear time-frequency distributions, the phase difference between the two frequencies can be computed by examining the phase of the cross-component time-frequency distribution of $s_{1}(t)$ and $s_{2}(t)$ [13]. In general, bilinear time-frequency representations provide higher frequency resolution, but suffer from the wellknown cross-term issue. For bilinear time-frequency distributions, careful design of data-dependent or fixed kernels for cross-term reduction should be performed so that the true auto-term points can be selected.

\section{SIMULATION RESULTS}

We first consider a single target scenario. The target swings around a center which is $5 \mathrm{~m}$ away from the radar with a maximum displacement of $1 \mathrm{~m}$. The observation period is one second. The two carrier frequencies are $900 \mathrm{MHz}$ and $1 \mathrm{GHz}$, respectively. The reflection coefficient is assumed to be a constant, regardless of the range. The input SNR is $0 \mathrm{~dB}$.

Figure 1(a) shows the magnitude of the STFT of $s_{1}(t)$. Due to the frequency resolution limitation, the STFT of $s_{2}(t)$ approximately coincides with that of $s_{1}(t)$. The sampling frequency is $1 \mathrm{kHz}$, and a 101-point Hanning window is used. As shown in Fig. 1(a), despite the low SNR level, the Doppler frequency signatures can be clearly identified in the STFT distributions. Figs. 1(b)-(d) show the range estimates using raw data, STFT phase, and the Doppler signature. It is evident from Fig. 1(b) that, because of the noise, the raw data based approach fails to provide meaningful range estimation. Significant improvement is achieved when using the phase information from the STFT. The best results are, however, obtained when applying the Doppler signature based approach. In this case, the range estimate error is very small.

Next, we consider two-target scenarios. We first consider a scenario where the Doppler frequencies of the two targets do not overlap. In this scenario, one person walks towards the radar, and the other walks away from the radar, at the same speed of $v=1 \mathrm{~m} / \mathrm{s}$. The targets were at the same initial range of $5 \mathrm{~m}$. The sampling frequency is $1 \mathrm{KHz}$. No noise is considered in this example.

Figure 2(a) shows the STFT magnitude at the carrier frequency 900 of MHz. A 501-point Hanning window is used, and the STFT is obtained using a 1024-point FFT transform. It is evident that the Doppler frequencies are separated in the time-frequency domain. From Fig. 2(b), it is observed that the raw data provides a constant range estimate, as the phase changes due to the movement of one target are cancelled by that of the other. On the other hand, as shown in Figs. 2(c)(d), STFT-based methods provide range estimates well matched to the true results, except small errors toward the edges due to the edge effect in Fig. 2(c).

In the next scenario, the Doppler frequencies of the two targets overlap. In this scenario, one walks away from the radar at a constant speed of $0.5 \mathrm{~m} / \mathrm{s}$, whereas the other walks away at a time-varying speed accelerating from $0 \mathrm{~m} / \mathrm{s}$ to $2 \mathrm{~m} / \mathrm{s}$ in the $5 \mathrm{sec}$ period. The initial ranges of the two targets are 4.5 $\mathrm{m}$ and $4 \mathrm{~m}$, respectively. Fig. 3(a) shows the STFT results at the carrier frequency of $900 \mathrm{MHz}$. The Doppler frequencies of the two targets are separated in the time-frequency domain for most of the observation period, but they overlap at around $t=$ $0.125 \mathrm{sec}$. As a result, poor range estimates occur around this moment when the STFT phase information is used, as seen in Fig. 3(c). This problem is overcome by using the Doppler 
signature, as deomnstarted by the range estimate in Fig. 3(d). For comparison, the use of raw data only yields a single range estimate in the middle position of the two targets (Fig. 3(b)).

\section{EXPERIMENTAL RESULTS}

To demonstrate the effectiveness of the proposed method in a real environment, laboratory experiments were conducted at Villanova University's Radar Imaging Lab (RIL). An I/Q dual-frequency radar with operation frequencies of 919.866MHz and $906.317 \mathrm{MHz}$ was employed. A 10-element Welded Yagi antenna was used for signal transmission and reception. The walls of the Lab were lined with electromagnetic absorbers to reduce ambient reflections. The received baseband data is preprocessed to remove clutter component near the DC frequency before the time-frequency analysis and range estimation are performed.

In the first experiment, two conducting spheres of $10^{\prime \prime}$ and 8 " diameter are mounted on separate linear positioners. One sphere remains stationary at the far end whereas the other moves back and forth over a $10 \mathrm{ft}(3.05 \mathrm{~m})$ range with a speed of $25 \mathrm{inch} / \mathrm{s}(0.635 \mathrm{~m} / \mathrm{s})$. Thus, it takes approximately $4.8 \mathrm{sec}$ for the sphere to travel the $10 \mathrm{ft}$ distance in one direction. The sampling frequency is $1 \mathrm{kHz}$ and the time duration of collected data is $20 \mathrm{sec}$. Fig. 4(a) shows the magnitude of the STFT of the baseband signal corresponding to carrier frequency of $919.866 \mathrm{MHz}$, where a 2001-point Hanning window is used. To obtain a high resolution in the frequency domain, the STFT is computed for 8192 frequency bins and only the frequency band of interest is shown in the figure. The estimated range using raw data is shown in Fig. 4(b), whereas that estimated from the STFT phase difference is shown in Fig. 4(c). Note that in this example the SNR is relatively high. As a result, the estimation result based on the raw data is relatively good, although some local variance is observed in the result. Such variance is not observed in Figs. 4(c)-(d) for the result based on time-frequency representations. Interestingly, range estimation results in Figs. 4(b)-(c) are affected by a phase distortion due to weak reflection from a computer rack near the antenna. Such effect is mitigated when the Doppler signature based approach is exploited, as shown in Fig. 4(d).

The second experiment has the same settings as the first one, but now both conducting spheres move with the same speed but in opposite directions. Fig. 5(a) shows the STFT magnitude of the baseband signal. When the raw data is used, as shown in Fig. 5(b), only the range of the sphere closer to the radar at a time is obtained as its return signal has dominate effect to the phase of the received signal. Time-frequency analysis based methods allow separation of the two targets. The STFT phase based method provides relatively good range

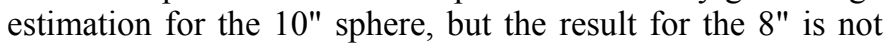
consistent. The Doppler signature based approach yields very robust range estimation for both targets.

\section{CONCLUSIONS}

We have presented a dual-frequency radar based timefrequency processing method for range-to-motion estimation in urban environments. The dual-frequency approach estimates the range of a moving target by estimating the phase difference of the signal returns at two closely spaced frequencies of operation. The performance of this scheme degrades significantly in high noise power level and with multiple moving targets. To overcome these shortcomings, we proposed the use of time-frequency signal representations where power can be localized in the time- frequency domain. Both simulation and experimental results were provided which clearly demonstrate the capability of the proposed method to accurately estimate the phase difference, thereby providing robust range-to-motion estimates in noisy environments as well as in the presence of multiple moving targets.

\section{ACKNOWLEDGEMENT}

The authors would like to thank Mr. Pawan Setlur at Villanova University for his assistance with real-data collection experiments.

\section{REFERENCES}

[1] Digest of the 2007 URSI North American Radio Science Meeting, Special Session on 'Through-Wall Imaging,' July 2007.

[2] Proceedings of the 2007 IEEE Workshop on Signal Processing Applications for Public Security and Forensics, Special Session on 'Through-the-Wall Imaging and Urban Sensing,' April 2007.

[3] S. E. Borek, "An overview of through the wall surveillance for homeland security," Applied Imagery and Pattern Recognition Workshop, Oct. 2005.

[4] Proceedings of the 2005 IEEE AP-S International Symposium, Special Session on 'Through-Wall Imaging and Sensing,' vol. 3B, pp. 317-341, July 2005.

[5] Proceedings of the 2004 IEEE AP-S International Symposium, Special Session on 'Through-Wall Imaging,' vol. 3, pp. 3059-3090, July 2004.

[6] D. D. Ferris, Jr. and N. C. Currie, "A survey of current technologies for through-the-wall surveillance," Proc. SPIE Sensors, and Command, Control, Communications, and Intelligence (C3I), Information, and Training Technologies for Law Enforcement, vol. 3577, pp. 62-72, San Diego, CA, Nov. 1998.

[7] W. D. Boyer, “A diplex, Doppler, phase comparison radar," IEEE Trans. on Aerospace and Navigational Electronics, vol. ANE-10, no. 3, pp. 2733, 1963.

[8] F. Ahmad, M. Amin, P. Setlur, "Through-the-wall target localization using dual-frequency CW radars," SPIE Symposium on Defense and Security, vol. 6201, Orlando, FL, April 2006.

[9] A. Lin and H. Ling, "Three-dimensional tracking of humans using very low-complexity radar," Electronics Letters, vol. 42, no. 18, pp. 10621064, Aug. 2006.

[10] F. Ahmad, M. G. Amin, and P. D. Zemany, "Performance analysis of dual-frequency $\mathrm{CW}$ radars for motion detection and ranging in urban sensing applications," Proc. SPIE Symposium on Defense and Security, Orlando, FL, April 2007.

[11] Y. Zhang, W. Mu, and M. G. Amin, "Subspace analysis of spatial timefrequency distribution matrices," IEEE Trans. Signal Processing, vol. 49, no. 4, pp. 747-759, April 2001.

[12] M. G. Amin, Y. Zhang, G. J. Frazer, and A. R. Lindsey, "Spatial timefrequency distributions: Theory and applications," in L. Debnath (ed.), Wavelets and Signal Processing, Boston, MA: Birkhauser, 2003.

[13] Y. Zhang, M. G. Amin, and F. Ahmad, "Range estimation of multiple targets using dual-frequency radars and time-frequency signal representations," Technical Report submitted to BAE Systems, May 2007.

[14] A. Jarrot, C. Ioana, C. Gervaise, and A. Quinquis, "A time-frequency characterization framework for signals issued from underwater dispersive environments," Proc. IEEE ICASSP 2007, Honolulu, HI, pp. III-1145-1148, April 2007. 

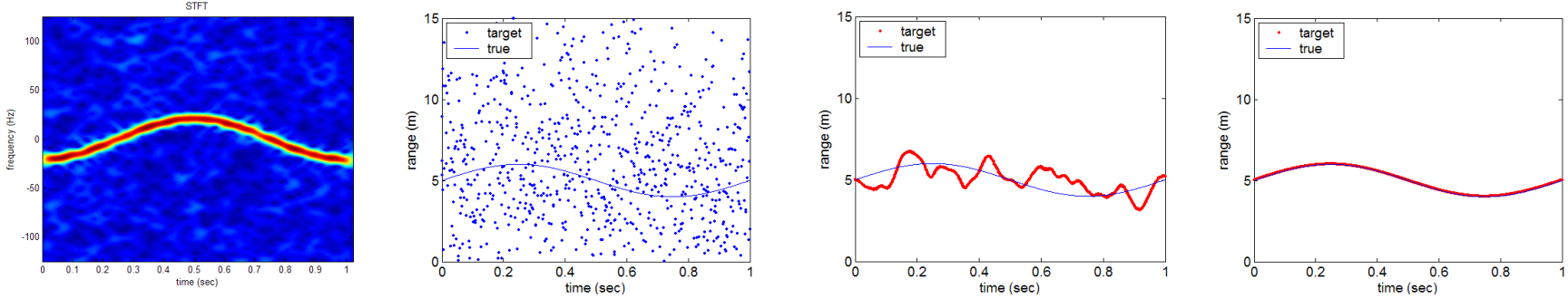

$\begin{array}{llll}\text { (a) STFT magnitude of } s_{1}(t) & \text { (b) Range estimate based on raw data } & \text { (c) Range estimate based on STFT phase } & \text { (d) Range estimate based on Doppler signature }\end{array}$ Figure 1 STFT and range estimation results of a single target case in the presence of noise $(\mathrm{SNR}=0 \mathrm{~dB})$.
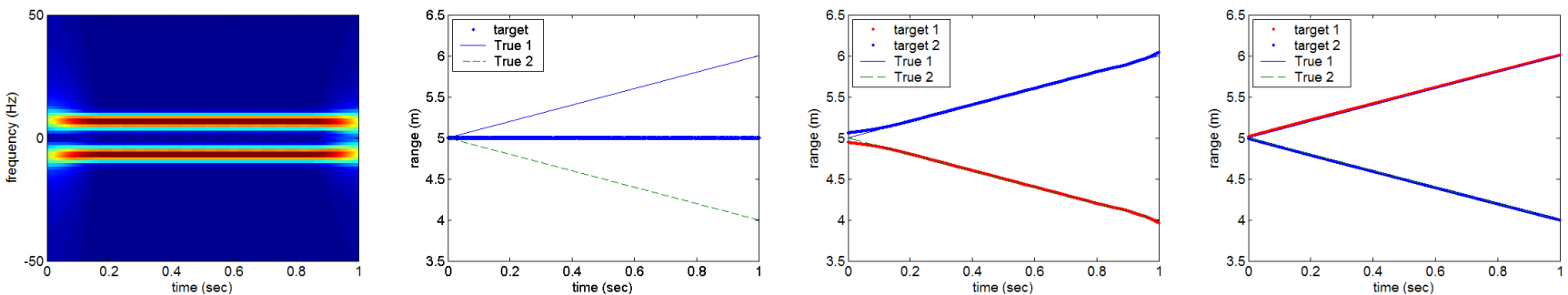

$\begin{array}{llll}\text { (a) STFT magnitude of } s_{1}(t) & \text { (b) Range estimate based on raw data } & \text { (c) Range estimate based on STFT phase } & \text { (d) Range estimate based on Doppler signature }\end{array}$

Figure 2 STFT and range estimates of two targets in the absence of noise. The targets have non-overlapping Doppler signatures in the time-frequency domain.
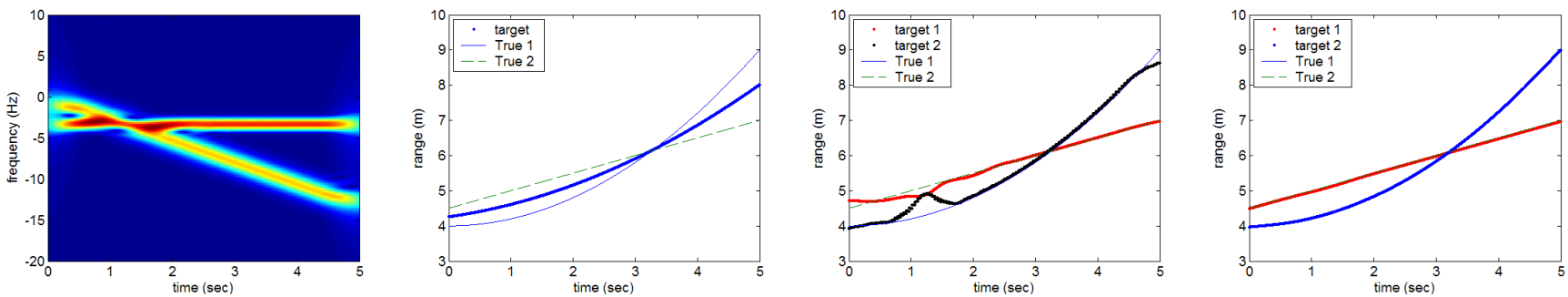

$\begin{array}{llll}\text { (a) STFT magnitude of } s_{1}(t) & \text { (b) Range estimate based on raw data } & \text { (c) Range estimate based on STFT phase } & \text { (d) Range estimate based on Doppler signature }\end{array}$

Figure 3 STFT and range estimates of two-targets in the absence of noise. The targets have overlapping Doppler signatures in the time-frequency domain.
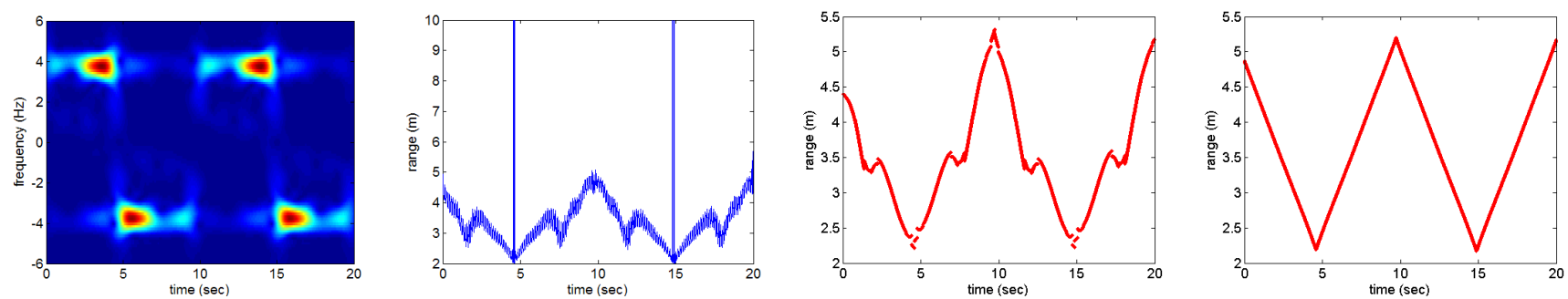

$\begin{array}{llll}\text { (a) STFT magnitude of } s_{1}(t) & \text { (b) Range estimate based on raw data } & \text { (c) Range estimate based on STFT phase } & \text { (d) Range estimate based on Doppler signature }\end{array}$

Figure 4 STFT and range estimation results of the first experiment where one conducting sphere moves back and forth.
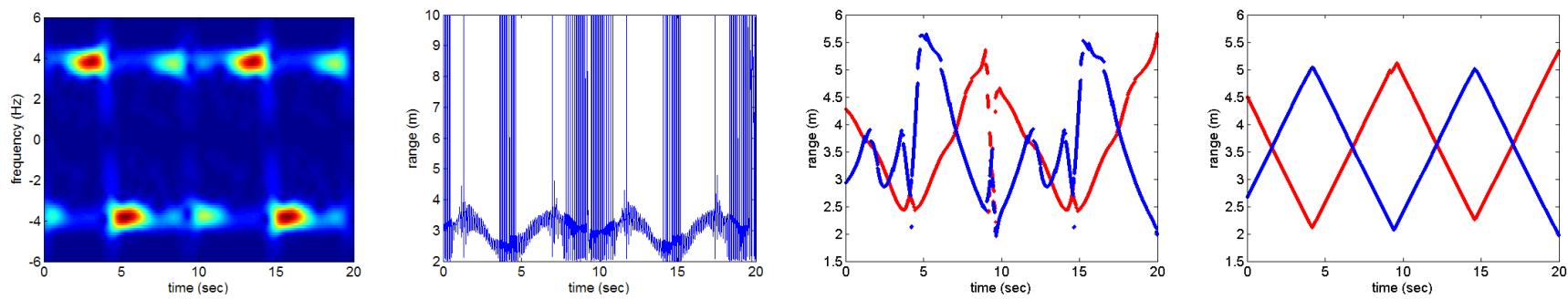

$\begin{array}{llll}\text { (a) STFT magnitude of } s_{1}(t) & \text { (b) Range estimate based on raw data } & \text { (c) Range estimate based on STFT phase } & \text { (d) Range estimate based on Doppler signature }\end{array}$

Figure 5 STFT and range estimates of the second experiment where both conducting spheres move with the same speed but in opposite directions. 